\title{
A NOTE ON FINITELY ADDITIVE SET FUNCTIONS
}

\section{RAMACHANDRAN}

\begin{abstract}
In this note, a counterexample to a conjecture of Yosida and Hewitt on finitely additive set functions is given.
\end{abstract}

Let $\boldsymbol{A}$ be a $\sigma$-field of subsets of a set $X$ and let $N$ be a proper subfamily of $\boldsymbol{A}$ which is closed under countable unions having the additional property that $N \in N, A \in A, A \subset N$ implies $A \in N$. Let $M$ denote the Banach space (under total variation norm) of all bounded, real valued finitely additive set functions on $(X, A)$ such that for every $N \in N$ and every $m \in M, m(N)=0$. Let $M^{*}=\{m \in M: m(A)=0$ or 1 for all $A \in A\}$. For each $A \in \boldsymbol{A}$ if $U_{\boldsymbol{A}}=\left\{m \in M^{*}: m(A)=1\right\}$ then $M^{*}$, equipped with the topology for which $\left\{U_{A}: A \in A\right\}$ is a base, is a compact Hausdorff space and $M$ is isometrically isomorphic to $C^{*}\left(M^{*}\right)$, the Banach space of all bounded real valued regular Borel measures on $M^{*}$.

Yosida and Hewitt [1] gave a necessary and sufficient condition for a member of $M$ to be countably additive, in terms of its isomorphic image in $C^{*}\left(M^{*}\right)$, and conjectured that if $M$ does not have any countably additive member at all, then every member $\bar{m}$ of $C^{*}\left(M^{*}\right)$ is confined to a closed nowhere dense $G_{\delta}$ in $M^{*}$. Lloyd [2] characterized purely finitely additive members of $M$ in terms of their isomorphic images in $C^{*}\left(M^{*}\right)$ from which it follows that if $M$ does not have any countably additive member at all then every member of $C^{*}\left(M^{*}\right)$ is confined to a countable union of closed nowhere dense $G_{\delta}$ sets in $M^{*}$, but offered no counterexample for the conjecture of Yosida and Hewitt. We give one below.

Let $X$ be the closed unit interval $[0,1], A$ Borel subsets of $X$ and $N$ first category Borel subsets of $X$. Since every countably additive Borel measure on $X$ lives in a first category Borel set, no member of $M$ is countably additive. But $M^{*}$ in this case is separable (see [3, p. 94]). Let $\left\{m_{1}, m_{2}, \cdots\right\}$ be a countable dense subset of $M^{*}$. If we define for each Borel subset $B$ of $M^{*}, \bar{m}(B)=\sum_{k=1}^{\infty} 2^{-k} I_{B}\left(m_{k}\right)$ where $I_{B}(\cdot)$ is the characteristic function of $B, \bar{m}$ is a countably additive Borel measure on $M^{*}$. Further $\bar{m}(B)=$ $\sup _{k} \bar{m}\left(B \cap\left\{m_{1}, \cdots, m_{k}\right\}\right)$ shows that $\bar{m}$ is regular, finite sets being compact

Received by the editors April 30, 1971.

AMS 1968 subject classifications. Primary 2813.

Key words and phrases. $\sigma$-field, finitely additive set function, purely finitely additive set function, regular Borel measure on a compact Hausdorff space. 
in $M^{*}$. Clearly $\bar{m}$ cannot be confined to any closed nowhere dense subset of $M^{*}$ and so the conjecture is false.

Acknowledgement. I thank Mr. K. P. S. Bhaskararao for pointing out Lloyd's result.

\section{REFERENCES}

1. K. Yosida and E. Hewitt, Finitely additive measures, Trans. Amer. Math. Soc. 72 (1952), 46-66. MR 13, 543.

2. S. P. Lloyd, On finitely additive set functions, Proc. Amer. Math. Soc. 14 (1963), 701-704. MR 28 \#4071.

3. R. Sikorski, Boolean algebras, 2nd ed., Ergebnisse der Mathematik und ihrer Grenzgebiete, Heft 25, Springer-Verlag, New York, 1964. MR 31 \#2178.

Research and Training School, Indian Statistical Institute, Calcutta, India 